\title{
Study on Adaptive Non-decimated Lifting Wavelet Packet Method for Failure Feature Extraction
}

\author{
SUN Ru-tian \\ Karamay Vacational \& Technical College \\ Karamay China \\ kzysrt@126.com
}

\begin{abstract}
This paper aims at removing the drawbacks of traditional wavelet transform in analyzing nonlinear reciprocating machinery failures, like energy losses and false frequencies. We combined the self-adapting strategy and non-decimating algorithm as an improvement in existing lifting scheme wavelet packet transform. Utilizing the Matrix Laboratory software, we tested and verified the performance of this new method in processing virtual datasets and actually collected signal. The spectrograms and tables in example and application indicate that this new method can obviously eliminate the false frequencies and extract the crucial characteristics in contaminated signals. And this property will enable it to be used in case of reciprocating and rotating machinery failure diagnosis.
\end{abstract}

Keywords- lifting wavelet packet; self-adaptive algorithm ; feature extraction; energy losses ; false frequencies

\section{INTRODUCTION}

Reciprocating machinery failures, especially the bearing wear and piston-cylinder wear, will generate periodic and non-stationary impact forces making the vibration signals nonlinear. Besides, lots of strong external interferences in collected signals make it difficult to improve the accuracy and validity of feature extraction . Because of its time-frequency localizing characteristics, wavelet transform has been widely applied in many fields during the past several decades. Some researchers employ it in the feature extraction for reciprocating machinery failures. However, energy loss caused by sampling, resolution determined by fixed wavelet function and scale cannot be adjusted as different failure signals and how to choose the fittest wavelet function still are the focus and nodus of current research.

The second generation wavelet packet has inherited the time-frequency localizing characteristics of traditional wavelet transform. And lifting scheme composed by nonlinear or space-adapting predictors and updaters is adopted to construct the calculation steps of it, getting rid of the Fourier transform, the dyadic algorithm and all the scaling conditions. It allows a faster and fully in-place calculation which can be accomplished just within the time domain .
In this paper, we combine non-decimating algorithm and Claypoole's self-adapting lifting scheme to create a new adaptive non-decimating lifting wavelet packet which can solve the problem of energy loss and becomes more corresponding with the characteristics of datasets to eliminate false frequency.

\section{ADAPTIVE NON-DECIMATED LIFTING ALGORITHM}

\section{A. Lifting Scheme Theory}

As known to all that the even subset and odd subset of engineering signals usually are highly correlated both in time (or space) and frequency so that if we know any one of them we can predict the other one within acceptable accuracy. Thus, in lifting scheme we firstly divide the original collected signal into two subsets, by using the "Lazy wavelet", which is called "split" session; then, in the "predict" session, we utilize the even subset to predict the odd subset and the prediction error is named as "detailed signal"; at last, we modify the even subset with the prediction error to make it approximate the odd subset, which is called "update" session and the modified even subset is defined as "approximated signal".

Suppose a set of real numbers, $s^{j}=\left\{s_{j, l}, 0 \leq l<2^{j}\right\}$ for $s^{j} \in R$, is the original signal. We decompose $s^{j}$ into two subsets: elements with even indexes are sorted into even subset even $^{j-1}$; while the rest is sorted into odd subset $o d d^{j-1}$, as follows:

$$
\begin{aligned}
& \text { even }^{j-1}=\left\{s_{j, 2 l}, 0 \leq l<2^{j}-1\right\} \\
& \text { odd }^{j-1}=\left\{s_{j, 2 l+1}, 0 \leq l<2^{j}-1\right\}
\end{aligned}
$$

Then, we predict the odd subset odd ${ }^{j-1}$ with even subset even $^{j-1}, P(g)$ is the predictor and the prediction error $d^{j-1}$ is the detailed signal.

$$
d_{j-1, l}=s_{j, 2 l+1}-P\left(s_{j, 2 l}\right)
$$

Particularly, if all elements of $s^{j}$ are constants, prediction error $d^{j-1}$ will be zero. 
Combining the prediction error $d^{j-1}$ and updater $U(g)$, we can modify the even subset even $^{j-1}$ into the approximated signal $s^{j-1}$.

$$
s_{j-1, l}=s_{j, 2 l}+U\left(d_{j-1, l}\right)
$$

Detailed signal $d^{j-1}$ can reflect the high frequency component of the original signal, whereas the approximated signal $s^{j-1}$ shows the low frequency component. Next procedures is taking the approximated signal $s^{j-1}$ as the original signal and repeating above three sessions in order until the prediction error is acceptable.

\section{B. Construction of Adaptive Predictor and Updater}

From the analysis above we know that all the characteristics of lifting scheme are completely determined by predictor and updater. Unique lifting scheme with specific properties can be achieved by designing corresponding predictor and updater. In this paper, we adopt Claypool's concept of equivalent filter for self-adapting lifting scheme to create a pair of new predictor and updater which are more corresponding with the characteristics of datasets.

Haworth and Sweden had proved that if the length of predictor $P(g)$ is $\mathrm{N}$, described as $P=[p(1) p(2) \cdots p(N)]^{T}$, and its equivalent filter is $G=[g(1) g(2) \cdots g(2 N-1)]^{T}$, then the rank of G's vanishing matrix is $\mathrm{M}(\mathrm{M}<\mathrm{N})$, and

$$
\left\{\begin{array}{c}
g(2 k)=\delta(k-N / 2) \\
g(2 k-1)=-p(k)
\end{array}\right.
$$

In the functions, $k=1, \cdots, N, \delta(k)$ is the Dirac function.

Thus, we design a Vandermonde matrix $\mathrm{V}$ as following conditions:

$$
\begin{gathered}
{[v]_{\mathrm{m}, \mathrm{n}}=n^{m} \cdot n=-(N-1), \cdots,(N-1) ; m=0, \cdots, M-1} \\
V \cdot G=0
\end{gathered}
$$

And we know that prediction error is

$$
d=\text { odd }-P(\text { even })
$$

Obviously, we would like to see that the differences between the original and approximated signal to be acceptable. That means

$$
{ }_{P}^{\min }\|d\|^{2}
$$

To reach this purpose, we should preserve one dimension of $P(g)$ to contain the characteristics of original dataset. Introducing the Lagrange coefficients and solving equations (2.7) and (2.9), we can get our target predictor $P(g)$ which can minimum the prediction error and also keep related with corresponding with the characteristics of original signal.
Based on the relationship between predictor and updater indexes, we assume updater $U(g)$ has $\tilde{N}$ indexes and victor $\mathrm{q}$ has $2 N-1$ indexes, and $\delta(k)$ is the Dirac function.

$$
\left\{\begin{array}{c}
q(2 i-1)=p(i) \\
q(2 i)=\delta(i-N / 2)
\end{array} i=1, \cdots, N .\right.
$$

Then, supposing $K=2 N+2 \tilde{N}-3$, we design another $\tilde{N} \times K$ Vandermonde matrix and matrix $H$,

$[W]_{m, n}=n^{m} . n=-N-\tilde{N}+2, \cdots, N+\tilde{N}-2 ; m=0, \cdots, \tilde{N}-1$.

$$
\left\{\begin{array}{c}
H[2 i-1: 2 i+2 N-3, i]=q \\
0, \text { else }
\end{array}\right.
$$

We can get our target updater $U(g)$ by solving function $W H U=[1,0,0 \cdots 0]^{T}$.

\section{Design of Non-decimated Lifting Wavelet Packet}

Although the adaptive lifting scheme overcomes the drawbacks of choosing wavelet function and scaling problem in traditional wavelet transform, the sampling frequency has been halved during the split session because it decomposes the original signal into low frequency component and high frequency component by low pass filter and high pass filter. Therefore, the resolution is reduced and part of the energy in original signal is lost. The high frequency component in low frequency band may generate frequency alias even produce false frequency.

Considering that, we introduce the non-decimating algorithm to avoid the sampling session. It can decompose the original signal at the same time-domain position within different scales and every frequency band has the same length of the original signal. The problem of energy loss will be resolved.

Assume $s_{l k}$ is a decomposed signal in the $k^{\text {th }}$ frequency band at $l^{\text {th }}$ layer, $s_{l(k-1)}$ and $s_{l k}$ are decomposed from $s_{(l-1)\left(\frac{k}{2}\right)}$, the detailed calculation formula are:

$$
\begin{aligned}
& s_{l(k-1)}(n)= \\
& s_{(l-1)\left(\frac{k}{2}\right)}(n)-\left[\begin{array}{l}
p_{1}^{[l]} s_{(l-1)\left(\frac{k}{2}\right)}\left(n-2^{l-1}(N+1)+1\right) \\
+p_{2}^{[l]} s_{(l-1)\left(\frac{k}{2}\right)}\left(n-2^{l-1}(N+1)+2\right)+\cdots+p_{2^{\prime} N}^{[l]} s_{(l-1)}\left(\frac{k}{2}\right) \\
\left(n+2^{l-1}(N-1)\right)
\end{array}\right]
\end{aligned}
$$

In the function, $k=2,4, \cdots, 2^{l}$ and $p^{[l]}$ is the nondecimated predictor of $s_{l(k-1)}$. 
$s_{l k}(n)=$
$s_{(l-1)\left(\frac{k}{2}\right)}(n)-\left[\begin{array}{l}u_{1}^{[l]} s_{l(k-1)}\left(n-2^{l-1}(\tilde{N}+1)+1\right)+u_{2}^{[l]} s_{l(k-1)}\left(n-2^{l-1}(\tilde{N}+1)+2\right) \\ +\cdots+u_{2^{\prime} \tilde{N}}^{l l]} s_{l(k-1)}\left(n+2^{l-1}(\tilde{N}-1)\right)\end{array}\right]$

And $k=2,4, \cdots, 2^{l}, u^{[l]}$ is the non-decimated updater of $s_{l k}$.

\section{EXAMPLE AND APPLICATION}

We can explain the method by an experiment. A signal contains several frequency components as following:

$f_{1}=20 \mathrm{~Hz} \quad, \quad f_{2}=60 \mathrm{~Hz} \quad, \quad f_{3}=90 \mathrm{~Hz}, f_{4}=120 \mathrm{~Hz}$, $f_{5}=180 \mathrm{~Hz}$.

With sampling frequency $f_{s}=400 \mathrm{~Hz}$, the collected signal is

$x(k)=\sin \left(\frac{2 \pi k f 1}{400}\right)+\sin \left(\frac{2 \pi k f 2}{400}\right)+\sin \left(\frac{2 \pi k f 3}{400}\right)+\sin \left(\frac{2 \pi k f 4}{400}\right)+\sin \left(\frac{2 \pi k f 5}{400}\right)$

If the lengths of predictor and updater are both 4 , the frequencies which are supposed to appear in every frequency band are shown in Table 1.

TABLE 1 THEORETICAL FREQUENCY COMPONENTS IN EACH FREQUENCY BAND

\begin{tabular}{llll}
\hline Decomposition layer & 1 th & 2 th & 3 th \\
\hline High frequency/Hz & 120,180 & 60,90 & - \\
\hline Low frequency/Hz & $20,60,90$ & 20 & 20 \\
\hline
\end{tabular}

While the actual processing results of traditional lifting scheme wavelet transform are shown in Figure1., Figure2. and Table 2. It is apparently that some false frequencies have showed up in the spectrogram, like $30 \mathrm{~Hz}$, $40 \mathrm{~Hz}, 80 \mathrm{~Hz}$, and $110 \mathrm{~Hz}$.

Then, we adopt the proposed method in this article with the same lengths of predictor and updater, as the Figure3. shows. The first impression of the spectrograms is that the false frequencies are removed and each major component in the original signal is properly distributed in corresponding frequency band. That is our expected primary result. In addition, the processed signals have the same length of original signal, which will remain equal energy as the original signal.

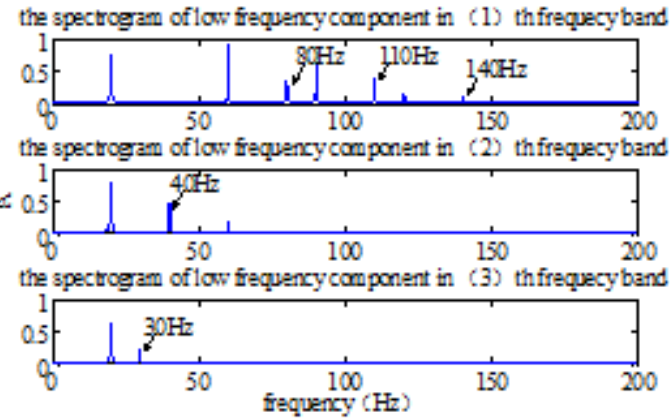

Figure I. The spectrograms of low frequency components in each frequency band using traditional lifting scheme wavelet transform

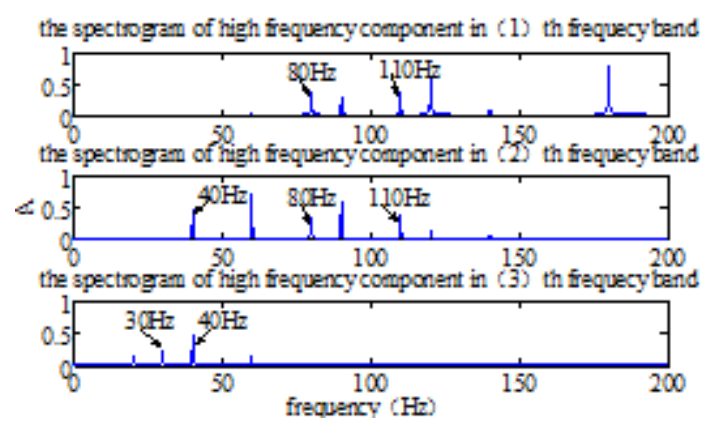

Figure II. The spectrograms of high frequency components in each frequency band using traditional lifting scheme wavelet transform
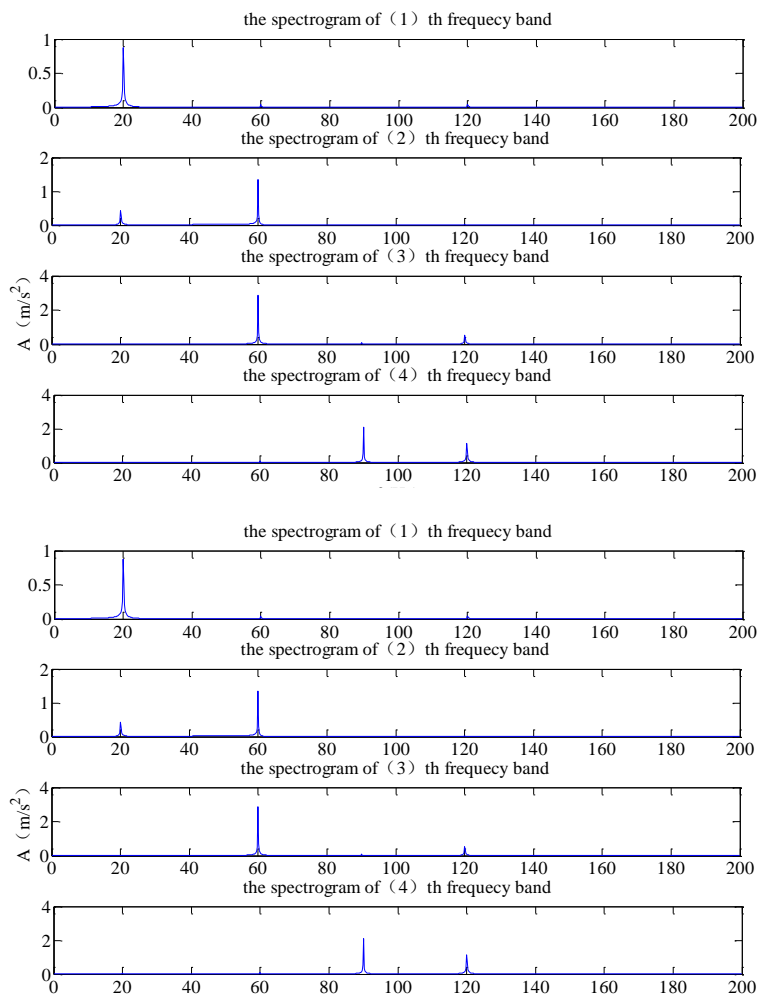

Figure III. the spectrograms of signals in each frequency band using proposed method 
TABLE 2 ACTUAL FREQUENCY COMPONENTS IN EACH FREQUENCY BAND USING TRADITIONAL WAVELET TRANSFORM

\begin{tabular}{llll}
\hline Decomposition layer & 1 th & 2th & 3 th \\
\hline High frequency/Hz & $\begin{array}{l}90,110,120, \\
180\end{array}$ & $\begin{array}{l}40,60,80,90, \\
110\end{array}$ & 40 \\
\hline Low frequency/Hz & $\begin{array}{l}20,60,80,90, \\
110,120\end{array}$ & 20 & 20,30
\end{tabular}

We take the DTY220MH-4.25 $\times 4$ reciprocating compressor in certain oil field as an example. It's driven by a motor through coupling. The motor's speed is $1500 \mathrm{rpm}$ and the period of the compressor is $0.04 \mathrm{~s}$. When the sampling frequency is $15000 \mathrm{~Hz}$ and the length of sample is 30720 , the collected normal signal and the signal on its second frequency band are shown in Figure4. and Figure5. So we see that in normal vibration signal there is an impulse every 0.04 second. It is caused by the reciprocating movement of piston. And several relatively smaller impulses appear in each period which is caused by valve movement and external inferences.

Later on, we collected another set of signal from the same compressor shown in Figure6. Firstly, the amplitude of vibration signal had increased by 60 percent, but we could not get more detailed information about what's different. So, we apply our proposed method to the collected signal, and then we could see that there was a major impulse in every $0.04 \mathrm{~s}$ which had completely submerged the former smaller impulses, as Figure7. shows. It's the typical phenomenon of piston wear. Thus, we supposed that there was something wrong with the piston of compressor. Later, the results of equipment maintenance verified our hypothesis.

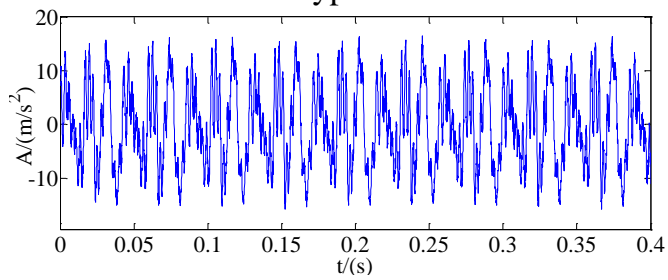

Figure IV. The vibration signal under normal operation condition

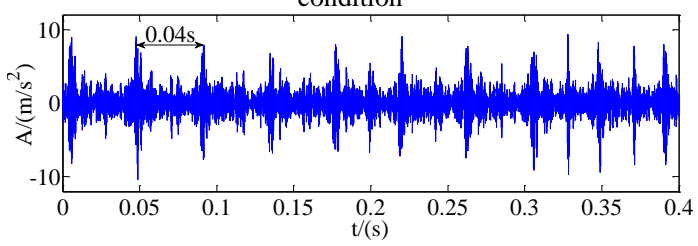

Figure V. proposed method

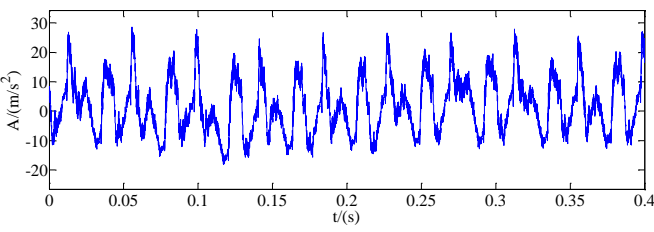

Figure VI. The vibration signal of piston wears

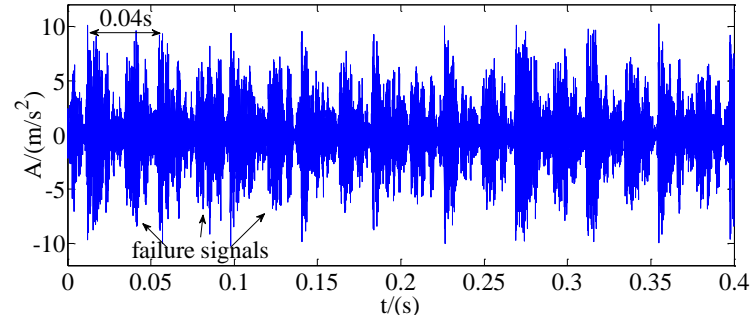

Figure VII. proposed method

The abnormal signal in 2th frequency band using

\section{CONCLUSION}

Two advanced algorithms, self-adapting strategy and non-decimating method, are adopted for the improvement of lifting scheme wavelet packet to extract failure features more efficiently. The experiment results of this new method suggest that false frequencies can be obviously eliminated and the energy of original signal are properly preserved. And the most imp 发 ortant thing is that crucial characteristics in contaminated signals are clearly revealed in every frequency band, which is easily received just from the spectrograms. We can conclude that this new method is much more outstanding than traditional wavelet packet transform, which enables it to cope with reciprocating and rotating machinery failures more effectively. Still, there are some aspects for us to explore, such as study on double adaptive lifting scheme transform, what is the proper variables for failure recognition with this method.

\section{REFERENCES}

[1] Jiang, H. K., Wang, Z. S., He, Z. J. (2008). Early identification of weak-signal fault features under very unfavourable environment using adaptive lifting scheme packet. Journal of North-western Poly technical University, 26(1), 99-102.

[2] Duan, C. D., Guo, Y. (2008). An envelope analysis approach for ball bearing based on lifting wavelet packet transform. Transactions of the Chinese Society for Agricultural Machinery, 39(5), 192-196

[3] Donoho, D. L. (1995). De-noising by soft-thresholding. IEEE Trans on Information Theory, 41(3), 613-627.

[4] Sweldens, W. (1996). The lifting scheme : A construction of second generation wavelets. SIAM J. Math. Anal, 29(2), 511-546.

[5] Claypoole, R. L. Jr. (1999). Adaptive wavelet transform via lifting. USA: Rice University, Texas.

[6] Daubechies, I., Sweldens, W. (1998). Factoring wavelet transform into lifting steps. J. Fourier Anal. App, 4(3), 247-269.

[7] Jiang, H. K., He, Z. J., Duan, C. D. (2006). Gearbox fault diagnosis using adaptive redundant lifting scheme. Mechanical Systems and Signal Processing, 20(8),1992-2006. 